ARTICLE

\title{
Cloning of wheat keto-acyl thiolase $2 B$ reveals a role of jasmonic acid in grain weight determination
}

\author{
Yun Chen 1,2,4, Yan Yan (1) 1,4, Tian-Tian Wu (10 1, Guo-Liang Zhang ${ }^{1}$, Huanran Yin ${ }^{3}$, Wei Chen ${ }^{3}$,
} Shuangshuang Wang ${ }^{1}$, Fang Chang $^{1} \&$ Jin-Ying Gou (iD) ${ }^{1 凶}$

Grain weight (GW) is one of the component traits of wheat yield. Existing reports have shown that multiple phytohormones are involved in the regulation of GW in different crops. However, the potential role of jasmonic acid (JA) remains unclear. Here, we report that triticale grain weight 1 (tgw1) mutant, with marked reductions in both GW and JA content, is caused by a premature stop mutation in keto-acyl thiolase $2 B(K A T-2 B)$ involved in $\beta$-oxidation during JA synthesis. KAT-2B overexpression increases $\mathrm{GW}$ in wild type and boosts yield. Additionally, KAT-2B compliments the grain defect in tgw1 and rescues the lethal phenotype of the Arabidopsis kat2 mutant in a sucrose-free medium. Despite the suppression of JA synthesis in tgw1 mutant, ABA synthesis is upregulated, which is accompanied by enhanced expression of SAG3 and reduction of chlorophyll content in leaves. Together, these results demonstrate a role of the JA synthetic gene $K A T-2 B$ in controlling $\mathrm{GW}$ and its potential application value for wheat improvement.

\footnotetext{
${ }^{1}$ State Key Laboratory of Genetic Engineering, MOE Key Laboratory for Biodiversity Science and Ecological Engineering, MOE Engineering Research Center of Gene Technology, Institute of Plant Biology, School of Life Sciences, Fudan University, Shanghai 200438, China. ${ }^{2}$ Institute of Metabolism and Integrative Biology, Fudan University, Shanghai 200438, China. ${ }^{3}$ National Key Laboratory of Crop Genetic Improvement and National Center of Plant Gene Research (Wuhan), Huazhong Agricultural University, Wuhan 430070, China. ${ }^{4}$ These authors contributed equally: Yun Chen, Yan Yan. ${ }^{凶}$ email: jygou@fudan.edu.cn
} 
s significant energy and protein resources for human beings, wheat is needed to increase another $50 \%$ from the current amount to feed the ever-growing world population by 2034 (International Wheat Yield Partnership). Wheat yield is mainly determined by three major dimensions: number of spikes per unit area, number of grains per spike, and grain weight $(\mathrm{GW})$. The first two dimensions determine the grain number (GN) per unit area of land, a dominant component for yield in environments with favorable growth conditions ${ }^{1}$. However, there is a trade-off between $\mathrm{GN}$ and $\mathrm{GW}^{2}$. Nevertheless, an increase of GW occurred in emmer wheat during domestication ${ }^{3}$. Therefore, GW enhancement is a feasible strategy to improve cereal yield depending on the environment ${ }^{1,4,5}$.

Grain size (GS) positively correlates with GW and has effectively promoted grain yield in rice ${ }^{6}$. Major quantitative trait loci and regulatory genes were cloned, which control the size and shape of rice grains through three major pathways, i.e., degradation of protein through the ubiquitin-proteasome process, signaling mediated by $\mathrm{G}$ protein/protein kinase, and plant phytohormone signal transductions mediated by auxin, cytokinin, and brassinosteroids ${ }^{7-16}$. Nevertheless, little studies reported the regulation of GS and GW by jasmonic acid (JA).

JA, as an essential plant hormone, fulfills critical roles in plant defense and development ${ }^{17}$. For example, JA confers pleiotropic effects on pollen grain and embryo developments ${ }^{18}$. In rice, downregulation of endogenous JA level by a 12-bp insertion in an enoyl-CoA hydrolase gene (NOG1) increased GN and grain yield ${ }^{19}$. A rice plastid-targeted lipase, EG2/OsJAZ1, has a critical regulatory role in spikelet development ${ }^{20}$. A wheat allene oxide cyclase (AOC) catalyzes a crucial step in the JA biosynthesis pathway and enhances salt tolerance in transgenic plants ${ }^{21}$. Besides, AOC is strongly induced in Leymus mollis, a wild relative of wheat grown along sea coasts, and can be introduced into wheat to enhance salt tolerance ${ }^{22}$. Beta-oxidation reaction catalyzes the chain shorting step in JA synthesis, fatty-acid degradation and conversion of indole-3-butyric acid (IBA) to indoleacetic acid (IAA). Ketoacyl thiolase (KAT) is an essential enzyme in the beta-oxidation reaction. The potential effect of JA on wheat GS and final yield is still unknown.

Here, we report the isolation of a triticale grain weight $1(\operatorname{tgw} 1)$ mutant from a tetraploid wheat EMS mutant library. We show that tgw1 produces smaller grains and accumulates less JA. This mutant contains a premature stop mutation in a 3-ketoacyl-CoA thiolase $(K A T-2 B)$ gene functioning in JA synthesis. The biological function of $K A T-2 B$ is confirmed by overexpression in wild type (WT), $\operatorname{tg} w 1$, and Arabidopsis kat 2 mutant. We show that $K A T-2 B$ overexpressors increase thousand kernel weight (TKW) and grain yield in field trials. These findings indicate that $K A T-2 B$ is valuable in dissecting the regulations of GS, GW, and GN-GW balance, as well as investigating JA's biological functions in wheat grain development.

\section{Results}

Phenotypic characterization of triticale grain weight 1 . We developed an EMS mutant library using tetraploid spring wheat (Kronos, WT hereafter) and screened for triticale grain weight (tgw) mutants with significant changes in TKW. tgw1 had shorter spikes than WT (Fig. 1a, b). At the full maturation stage, tgw1 grains exhibited evident reductions in length and width compared with WT (Fig. 1c, d). The average GW of $\operatorname{tgw} 1$ was $26 \pm 0.6 \mathrm{mg}$, which was significantly lower than WT control $(39 \pm 0.6 \mathrm{mg})$ in a growth chamber (Fig. 1e). Microscopical examination revealed that the endodermal cells of tgw1 were significantly narrower than those of the WT line (Fig. 1f, g).

Compared with WT, $\operatorname{tg} w 1$ showed a significant reduction in the leaf area (Fig. 1h). However, there was no difference in the average area of cells in the leaf (Fig. 1i, Supplementary Fig. 1), suggesting a potential reduction in cell numbers instead. Furthermore, there is a significant reduction in the mutant's chlorophyll contents than WT (Fig. 1j). Therefore, both changes in the leaf area and chlorophyll content were unfavorable for the pool's energy source, contributing to the GW change.

Molecular cloning of TGW1. Bulked segregant analysis (BSA) identified potential correlations between phenotype and genotype in $\mathrm{BC}_{1} \mathrm{~F}_{2}$ generation. A total of $40 \mathrm{Giga}$ data were sequenced from three samples (S1, S2, S3), each containing eight individual homozygous $\mathrm{BC}_{1} \mathrm{~F}_{2}$ plants. Only two nonsynonymous homozygous SNPs, located in TraesCS6B02G432600 and TraesCS6B02G414800, respectively, were detected in all three samples (Fig. 2a).

To investigate which of the two genes may be the candidate TGW1 gene, we first checked their expression levels in wheat according to the digital Northern data extracted from public microarray results (GSE12508 in NCBI). The expression of TraesCS6B02G414800 was low in most tissues (Supplementary Fig. 2). The other gene, TraesCS6B02G432600, annotated to encode KAT-2, had a signal of over 20 fold higher than that of TraesCS6B02G414800 in all the analyzed tissues (Supplementary Fig. 2).

Based on 71 RNA-seq studies in different tissues at various developmental stages (http://bar.utoronto.ca/efp_wheat/cgi-bin/ efpWeb.cgi), the expression of KAT-2B (TraesCS6B01G432600) was higher than KAT-2A (TraesCS6A01G392400) (Fig. 2b, Supplementary Data 1). There was a CAA to TAA mutation in the $12^{\text {th }}$ exon of $K A T-2 B$ in $\operatorname{tg} w 1$ (Fig. 2c), which introduced a premature stop codon $\left(\mathrm{Q} 364^{*}\right)$ at a position with 92 amino acids to the $\mathrm{C}$ terminal end of KAT-2 (Fig. 2d). Based on the KAT-2's structure, two out of the three active sites (H-407 and N-447) are critical for KAT-2 activity located after Q364 ${ }^{23}$. Hence, the Q364* mutation should have an inhibitory effect on the biochemical activity of KAT-2. In Arabidopsis, kat2 mutant also showed reduced reproductive success, with substantially seed less yield and single seed weight ${ }^{24,25}$.

KAT2 catalyzes an essential step in $\beta$-oxidation reaction to reduce carbon chain length and produce acetyl CoA during fattyacid degradation and the biosynthesis of two crucial plant hormones, IAA and $\mathrm{JA}^{24,25}$. To explore potential metabolite changes between WT and $\operatorname{tg} w 1$, we quantified contents of fatty acids by GC/MS, IAA by LC/MS, and JA by enzyme-linked immunosorbent assay (ELISA). No significant differences showed up in free fatty acids profiles or IAA contents between $\operatorname{tgw} 1$ and WT (Supplementary Fig. 3). However, the JA content in the tgw1 mutant was $50 \%$ less than that of WT, a significant change (Fig. 2e). The level of oxidized lipid was also lower in $\operatorname{tg} w 1$ than in WT (Supplementary Fig. 4).

In the tgw1 mutant, a gene annotated as a cell number regulator (CNR6) significantly decreased at the expression level (Fig. 2f), which correlated to the cell number changes in the $\operatorname{tgw} 1$ mutant. Upon JA treatment, the expression of CNR6 significantly increased in seedling leaves (Fig. 2g). On the contrary, the JA inhibitor application, sodium diethyldithiocarbamate (DIEC), suppressed the expression of CNR6 in a time-dependent manner (Fig. 2h). Therefore, both exogenous JA and inhibitor treatment suggested JA's role in regulating CNR6 expression, as shown in the $\operatorname{tg} w 1$ mutant.

Moreover, the tgw1 mutant produced smaller anthers with fewer pollen grains (Supplementary Fig. 5a), many of which were defective (Supplementary Fig. 5b-d). Quantitative analysis showed that the defective pollen grains of $\operatorname{tg} w 1$ (11.8\%) was significantly higher than that of WT control (4\%) (Supplementary 

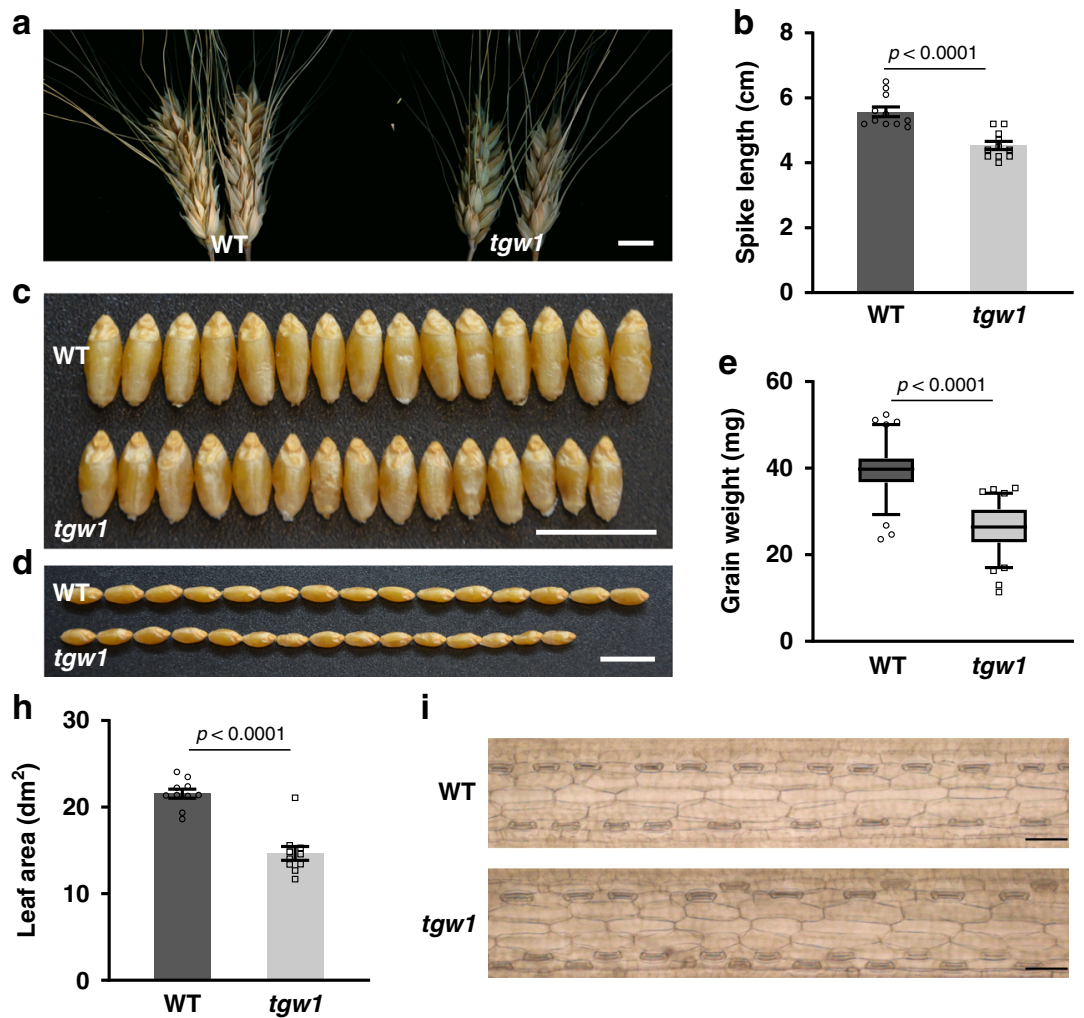

f
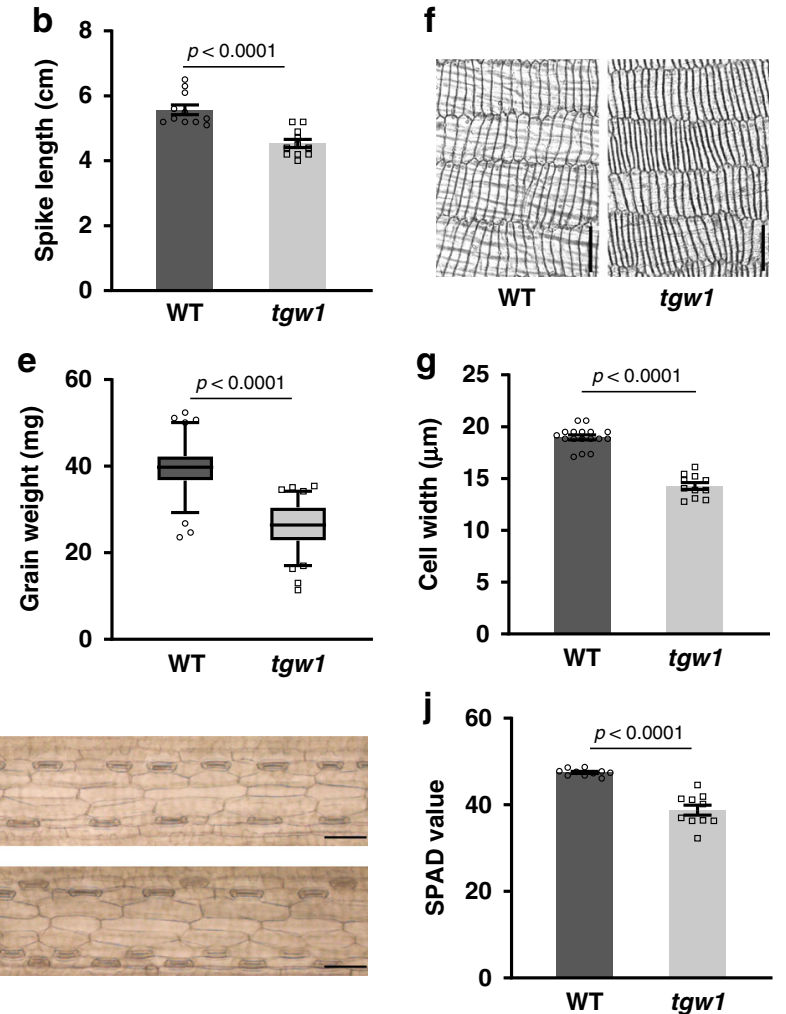

Fig. 1 Identification and characterization of tgw1. a Representative images of main tiller spikes from WT or tgw1. Scale bar $=1 \mathrm{~cm}$. $\mathbf{b}$ Average length of main spikes from WT and tgw1. $n=11$. c-d Comparison of width and length of mature grains from WT or tgw1. Scale bar $=1 \mathrm{~cm}$. e Average grain weight in WT and tgw1. $n=80$. $\mathbf{f}$ Representative images of grain endodermal cells in WT and tgw1. Scale bar $=100 \mu \mathrm{m}$. $\mathbf{g}$ Average width of grain endodermal cell in WT $(n=17)$ and tgw1 $(n=11)$. $\mathbf{h}$ The average area of flag leaves in WT and tgw1 mutant. $n=10$. $\mathbf{i}$ Leaf epidermal cells in WT or tgw1 mutant. Scale bar $=$ $100 \mu \mathrm{m}$. j Average chlorophyll units in flag leaves from WT and tgw1 mutant. $n=10$. Data in e are represented as boxplots. The boxplots indicate median (middle line), $25^{\text {th }}, 75^{\text {th }}$ percentile (box), and $5^{\text {th }}$ and $95^{\text {th }}$ percentile (whiskers) as well as outliers (single points). Data in $\mathbf{b}, \mathbf{e}, \mathbf{g}, \mathbf{h}$, and $\mathbf{j}$ are represented as mean \pm SEM, and $p$ values are indicated by two-tailed unpaired $t$ test. Results in $\mathbf{f}$ and $\mathbf{i}$ are representative of three independent samples. Source data are provided as a Source Data file.

Fig. 5e). The above changes were consistent with early reports that JA played an essential role in the development of pollens in model plants ${ }^{18,26}$. These results suggested that wheat $K A T-2 B$ is very likely involved in the biosynthesis of JA.

Validation that $K A T-2 B$ is TGW1. First, we introduced a $K A T$ $2 B$ gene (Pro ${ }_{U b i}: K A T-2 B$-Flag) into the WT wheat line by genetic transformation. In a quantitative analysis, the $K A T-2 B$ expression level was higher in the transgenic plants than WT (Fig. 3a). At the protein level, KAT-2 protein showed an apparent reduction in tgw 1 but accumulated at a higher level in the transgenic overexpression lines (Fig. 3b). KAT-2A could very likely encode the residual KAT-2 in the $\operatorname{tg} w 1$ mutant, expressed at a relatively lower level (Fig. 2b). In seedlings, JA content was significantly higher in the transgenic line KAT-2B O.E. $3^{\#}$ (Fig. 3c). In transgenic line 4 with no change in seedlings, the JA content significantly increased in leaves treated upon pathogen treatment (Supplementary Fig. 6).

Next, the expression level of CNR6 in the transgenic lines was evaluated by real-time quantitative PCR (qRT-PCR). In KAT-2B O.E. $3^{\#}$, CNR6 expressed at a significantly higher level than WT (Fig. 3d). Consistently, the flag leaf area increased by $14.5 \%$ and $9.2 \%$ in KAT-2B O.E. $3^{\#}$ and KAT-2B O.E. $4^{\#}$, respectively (Fig. 3e). In the chlorophyll content, both lines showed increases of $9.5-11.1 \%$, which were very significant compared with WT (Fig. 3f). Overall, both leaf area and chlorophyll content indicated that the photosynthesis capacity should increase in the KAT-2B transgenic lines. As a result, the transgenic grains displayed a definite increase in width compared with WT but had no apparent difference in length (Fig. 3g). Contrary to $\operatorname{tg} w 1, K A T-2 B$ transgenic lines had an average $\mathrm{GW}$ of $45 \mathrm{mg}$, which was significantly higher than WT (Fig. 3h).

The agricultural traits of $K A T-2 B$ transgenic, $\operatorname{tg} w 1$, and WT were evaluated in the field in June 2020. Consistent with the leaf defects in $t g w 1$, grain number per spike (GNS) and grain weight per spike (GWS) in tgw1 reduced by $7.3 \%$ and $18.3 \%$, respectively, significantly lower than that of WT (Table 1). However, GNS in the KAT-2B O.E. transgenic line main spikes was reduced by $2.4-9.3 \%$ (Table 1 ), reflecting a GN-GW trade-off reported previously ${ }^{2}$. In the transgenic lines, the grain yield per plant increased by $18 \%$ to $28 \%$ than that of WT. The transgenic event of $K A T-2 B$ in wheat led to an over $15 \%$ increase in the final yield per unit area in the two transgenic lines (Table 1). These data were consistent with the field trial in June 2019 (Supplementary Table 1). These results agreed with the leaf area and chlorophyll contents changes, as the source limitation to GW in modern wheat cultivars ${ }^{26}$.

To confirm that KAT-2B $\mathrm{B}^{264^{*}}$ is $\operatorname{tg} w 1$, we crossed $K A T-2 B$ transgenic plant with $\operatorname{tg} w 1$ to overexpress wild-type $K A T-2 B$ into $\operatorname{tg} w 1$ (KAT-2B/tgw1). All four complementation lines accumulated variable KAT-2B (Fig. $3 \mathrm{i}, \mathrm{j}$ ). The chlorophyll content was also restored in the complementation lines (Fig. 3k). The grains displayed apparent increases in width compared with $\operatorname{tg} w 1$ (Fig. 31). Based on GW, complete complementation of the $\operatorname{tgw} 1$ phenotype was observed in all four lines, demonstrating that $K A T-2 B$ transgene successfully rescued the GW defect in $\operatorname{tg} w 1$ 
a

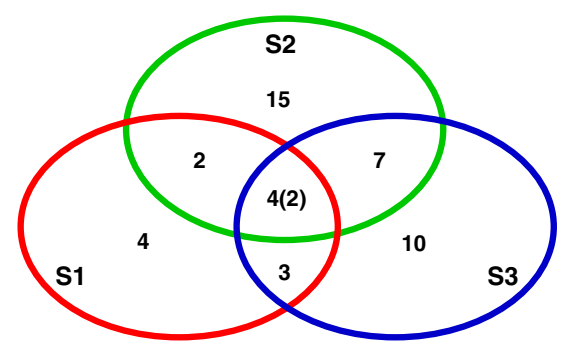
$\underset{(K A T-2 B)}{\operatorname{TGW1}}$

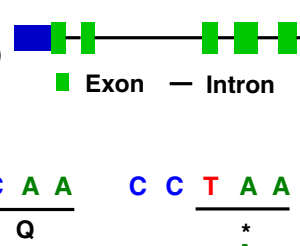

d
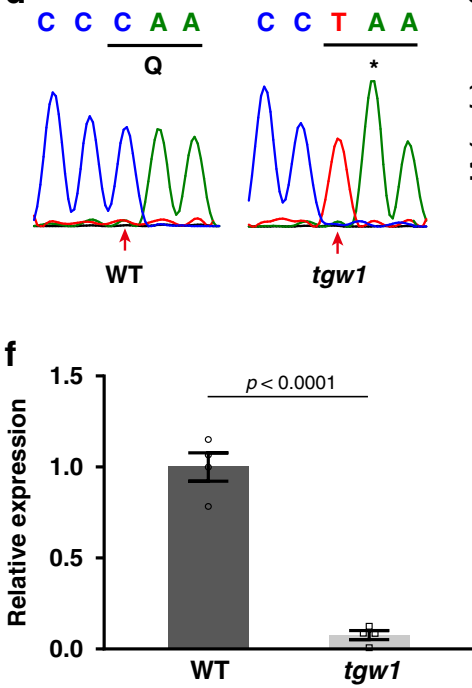
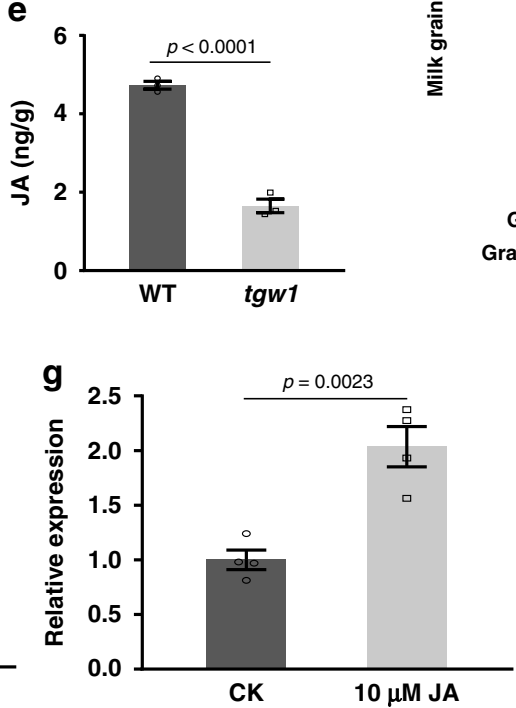

b
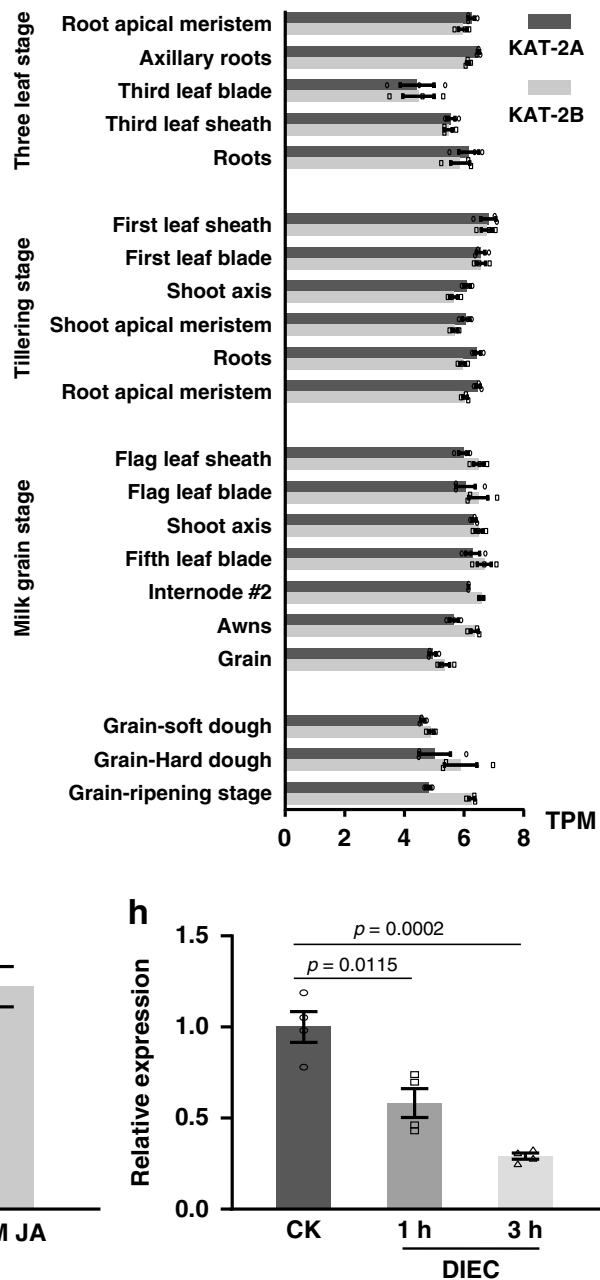

Fig. 2 Molecular cloning of TGW1. a Venn diagram to show distributions of homozygous SNPs in three groups of homozygous $\mathrm{BC}_{1} \mathrm{~F}_{2}$ mutants. $\mathrm{S} 1$ to $\mathrm{S}_{3}$ represented three groups of homozygous $B_{1} F_{2}$ mutants. Each number represents numbers of homozygous SNPs. Only two nonsynonymous homozygous SNPs presented in three groups. b The expression of KAT-2B (TraesCS6B01G432600) and KAT-2A (TraesCS6A01G392400) in different tissues at various developmental stages. $n=3$ independent replicates. $\mathbf{c}$ Genomic structure of TGW1 (KAT-2B) gene. The red triangle points to the position of the point mutation in tgw1. d Verification of the SNP in the KAT-2B (TGW1) gene in WT and tgw1 by sequencing. e Contents of JA in leaves of WT and tgw1. $n=3$. f Relative expression level of Cell Number Regulator 6 (CNR6) in WT and tgw1 mutant. $n=4$. $\mathbf{g}$ The expression level of CNR6 upon $10 \mu M$ JA treatments in WT. $n=4$. $\mathbf{h}$ The expression level of CNR6 upon $200 \mu$ M JA inhibitor DIEC treatments in WT. $n=4$. Data are represented as mean \pm SEM in $\mathbf{b}$, and $\mathbf{e}-\mathbf{h}$. $p$ values are indicated by two-tailed unpaired $t$ test in $\mathbf{e}-\mathbf{h}$. Source data underlying Figs. $2 a, b$, e-h are provided as a Source Data file.

(Fig. 3m). Thus, both BSA (Fig. 2a) and complementation results validated $K A T-2 B$ as $T G W 1$.

Molecular characterization of $K A T-2 B$. After validating the function of $K A T-2 B$ by genetics, we then investigated more basic molecular characteristics of $K A T-2 B$. As shown by RT-PCR, $K A T-2 B$ highly expressed in most tissues except embryo (Fig. 4a), in agreement with the public microarray data (Supplementary Data 1).

To examine the biochemical activity of KAT-2B, we purified KAT-2B recombinant protein. Thiolase activity was readily detected for recombinant KAT-2B using acetoacetyl CoA and $\mathrm{CoA}$ as substrates, representing a typical carbon chain shortening step in $\beta$-oxidation (Fig. 4b). Moreover, the specific activity of KAT-2B was activated by some monovalent cations $\left(\mathrm{K}^{+}\right.$and $\mathrm{Na}^{+}$) but inhibited by certain divalent cations (e.g., $\mathrm{Mg}^{2+}$ ) in vitro (Supplementary Fig. 7a). The recombinant KAT-2B protein preferred alkaline conditions ( $\mathrm{pH}$, ranging from 7.5 to 9 ) (Supplementary Fig. 7b).
To further test the involvement of $K A T-2 B$ in the $\beta$-oxidation process, we fused $K A T-2 B$ with $G F P$ in a transgenic vector (Pro $_{355}$ : GFP-KAT-2B) and transiently co-expressed it with a peroxisome marker, AKT1-mCherry ${ }^{27}$, in tobacco leaves. Under a confocal microscope, GFP-KAT-2B co-localized with AKT1mCherry in leaf epidermal cells (Supplementary Fig. 8). In the protoplast prepared from mesophyll cells, GFP-KAT-2B fusion protein also co-localized with AKT1-mCherry (Fig. 4c). These data suggested that KAT-2B performed its biological function in the peroxisome, the organelle where the $\beta$-oxidation reaction occurs.

The role of wheat KAT-2 in $\beta$-oxidation was also validated by complementation of Arabidopsis kat2 mutant, which cannot convert endogenous lipids into acetyl CoA to produce energy; therefore is lethal in $1 / 2$ MS medium without sucrose as the solo exogenous energy resource ${ }^{28}$. KAT-2 homeologs from the A and $\mathrm{B}$ genomes of wheat were transformed into Arabidopsis kat2 homozygous mutant (Supplementary Fig. 9). As expected, the resultant KAT-2 Arabidopsis transgenic lines grew well on the sucrose-free medium (Fig. $4 \mathrm{~d}$ ), indicating that wheat $K A T-2$ in 

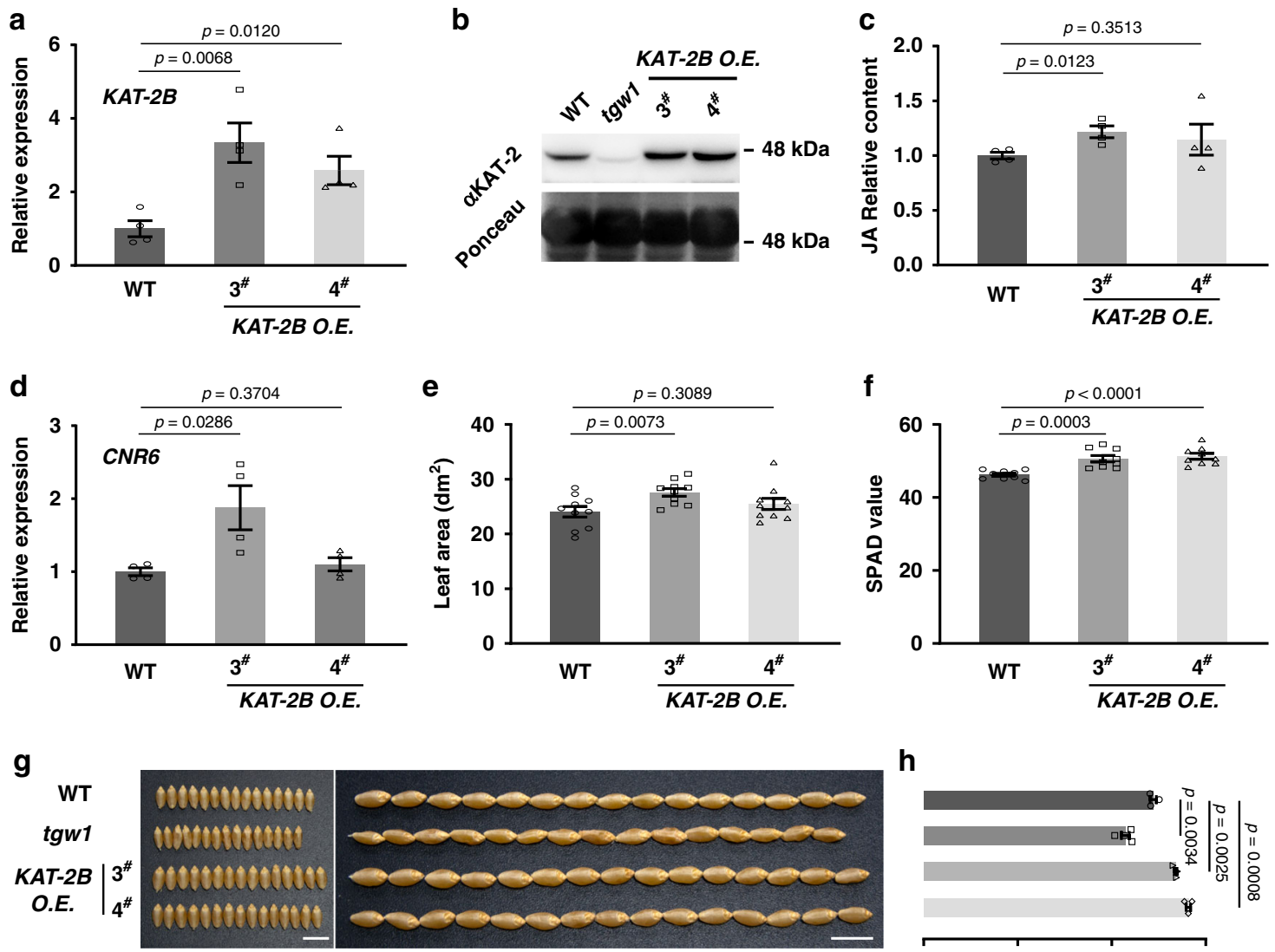

h
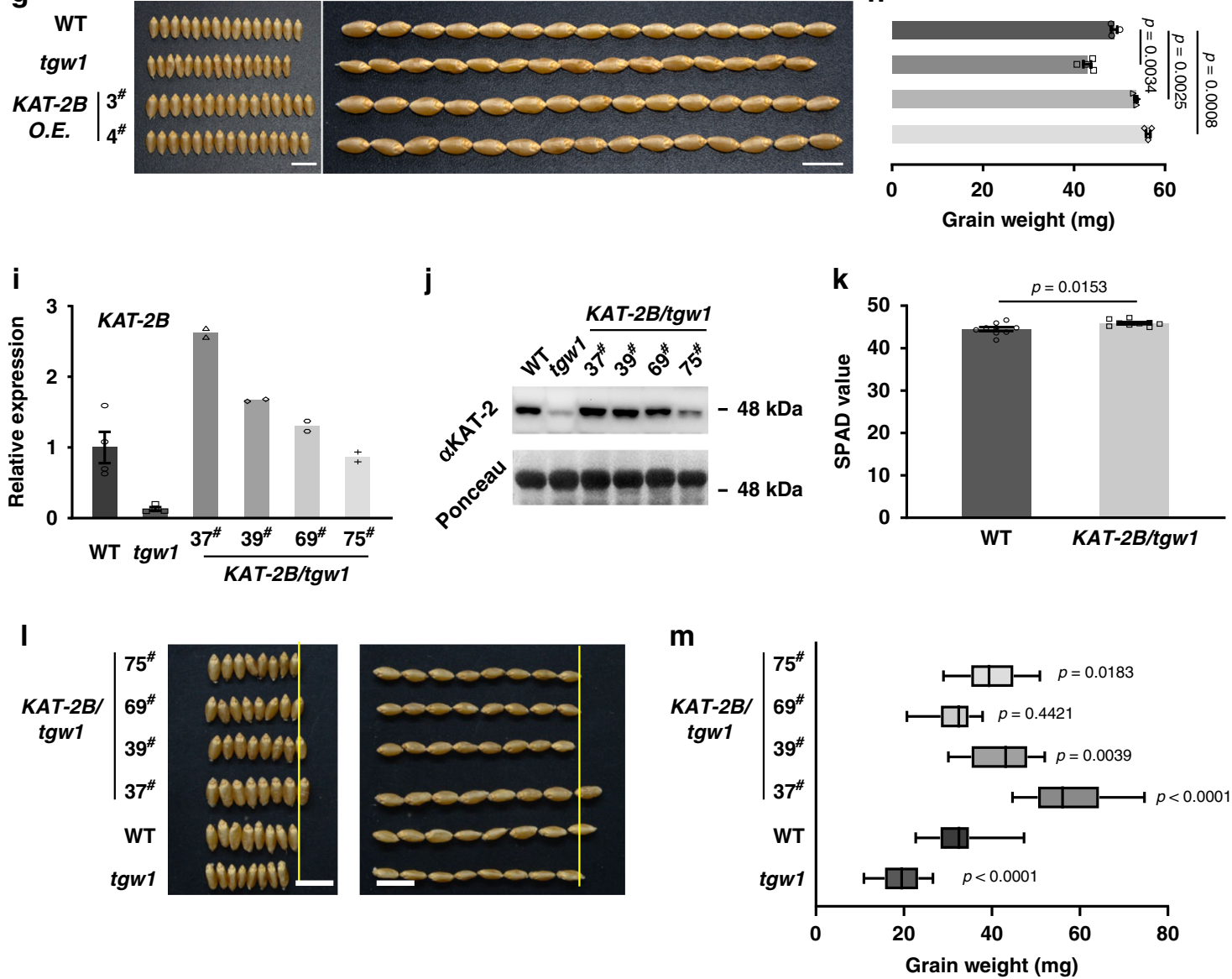

both $\mathrm{A}$ and $\mathrm{B}$ genomes could entirely rescue the $\beta$-oxidation defect in Arabidopsis kat2 mutant.

Plant hormone biosynthesis changes in tgw1. The expression change of CNR6 could partially contribute to the leaf area changes in the $\operatorname{tg} w 1$ mutant line. Nevertheless, there is no clue for the changes in the chlorophyll contents in the tgw1 mutant. To investigate processes involved in the regulation of GW and other

traits, we compared the transcriptome of the three groups of plants in tgw1 $\mathrm{BC}_{1} \mathrm{~F}_{2}$ generation with WT (Fig. 5a, Supplementary Data 2).

Interestingly, the expression levels of several genes in phytohormone metabolism altered in tgw1, including the suppression of 14 genes in JA synthesis and one gene in gibberellic acid (GA) biosynthesis (Table 2). The former was consistent with the change of oxidized lipids in tgw1 
Fig. 3 The biological function of $\boldsymbol{K A T - 2 B}$ in transgenic plants. a Comparison of $K A T-2 B$ expression level by qRT-PCR in WT, and transgenic wheat. $n=4$. b Western blot analysis of KAT-2. Ponceau staining of RuBisCO serves as a loading control. c Relative quantification of JA in WT and transgenic wheat. $n=$ 4. WT was set to 1. $\mathbf{d}$ Expression level of CNR6 in transgenic wheat. $n=4$. e-f Comparison of average flag leaf areas e and chlorophyll content $\mathbf{f}$ in WT and transgenic wheat. $n=10$ e. $n=9 \mathbf{f}$. $\mathbf{g}$ Comparison of grains from in WT, tgw1 mutant, and KAT-2B transgenic lines. Scale bar $=1 \mathrm{~cm}$. $\mathbf{h}$ Average weight of grains from WT $(n=3)$, tgw1 mutant $(n=4)$, and transgenic lines $(n=3)$. i-j Comparison of KAT-2B expression (i) and protein (j) levels in tgw1 mutant $(n=4), \mathrm{WT}(n=4)$, and complementation lines. $\mathbf{k}$ Comparison chlorophyll contents in KAT-2B/tgw1 and tgw1 mutant lines. $n=9$. I Comparison of grains from in $K A T-2 B / \operatorname{tg} w 1, W T$, and tgw1 mutant lines. Scale bar $=1 \mathrm{~cm}$. $\mathbf{m}$ Comparison of average grain weight in $K A T-2 B / \operatorname{tg} w 1, W T$, and tgw1 mutant. $n=12$. Results in $\mathbf{b}$ and $\mathbf{j}$ are representative of two independent experiments. In $\mathbf{m}$ data are represented as boxplots. The boxplots indicate median (middle line), $25^{\text {th }}, 75^{\text {th }}$ percentile (box) and $5^{\text {th }}$ and $95^{\text {th }}$ percentile (whiskers). Data from complementation lines in $\mathbf{i}$ are represented as mean from two technical replicates. Data in $\mathbf{a}, \mathbf{c}-\mathbf{f}, \mathbf{h}, \mathbf{i}(\mathrm{WT}, \operatorname{tg} w 1), \mathbf{k}$ and $\mathbf{m}$ are represented as mean \pm SEM from independent biological replicates, and $p$ values are indicated by twotailed unpaired $t$ test. Source data underlying Fig. $3 a-k, m$ are provided as a Source Data file.

Table 1 Agricultural traits of WT, tgw1, and KAT-2B overexpression lines in the field in June 2020.

\begin{tabular}{|c|c|c|c|c|}
\hline & WT & $\operatorname{tgw1}$ & KAT-2B O.E. $3^{\#}$ & KAT-2B O.E. $4^{\#}$ \\
\hline $\mathrm{TN}$ & $\begin{array}{l}3.59 \pm 0.78 \\
n=63\end{array}$ & $\begin{array}{l}3.55 \pm 0.59 \\
p=0.7791, n=42\end{array}$ & $\begin{array}{l}5 \pm 1.34 \\
p<0.0001, n=20\end{array}$ & $\begin{array}{l}4.77 \pm 1.02 \\
p<0.0001, n=22\end{array}$ \\
\hline SPS & $\begin{array}{l}17.39 \pm 1.03 \\
n=88\end{array}$ & $\begin{array}{l}16.07 \pm 1.03 \\
p<0.0001, n=63\end{array}$ & $\begin{array}{l}17.13 \pm 1.6 \\
p=0.2705, n=40\end{array}$ & $\begin{array}{l}17.67 \pm 1.11 \\
p=0.1703, n=39\end{array}$ \\
\hline $\mathrm{SPL}(\mathrm{cm})$ & $\begin{array}{l}7.21 \pm 0.54 \\
n=88\end{array}$ & $\begin{array}{l}6.29 \pm 0.52 \\
p<0.0001, n=61\end{array}$ & $\begin{array}{l}7.48 \pm 0.55 \\
p=0.0106, n=40\end{array}$ & $\begin{array}{l}7.41 \pm 0.48 \\
p=0.0509, n=40\end{array}$ \\
\hline GNS & $\begin{array}{l}44.78 \pm 5.76 \\
n=87\end{array}$ & $\begin{array}{l}41.49 \pm 6.66 \\
p=0.0022, n=61\end{array}$ & $\begin{array}{l}43.7 \pm 4.54 \\
p=0.2971, n=40\end{array}$ & $\begin{array}{l}40.62 \pm 4.35 \\
p=0.0001, n=37\end{array}$ \\
\hline GWS (g) & $\begin{array}{l}2.18 \pm 0.33 \\
n=53\end{array}$ & $\begin{array}{l}1.78 \pm 0.34 \\
p<0.0001, n=63\end{array}$ & $\begin{array}{l}2.31 \pm 0.24 \\
p=0.0385, n=38\end{array}$ & $\begin{array}{l}2.16 \pm 0.21 \\
p=0.7565, n=32\end{array}$ \\
\hline TKW (g) & $\begin{array}{l}49.37 \pm 0.97 \\
n=7\end{array}$ & $\begin{array}{l}45.9 \pm 0.34 \\
p<0.0001, n=5\end{array}$ & $\begin{array}{l}51.43 \pm 0.39 \\
p=0.0012, n=5\end{array}$ & $\begin{array}{l}51.35 \pm 0.73 \\
p=0.0033, n=5\end{array}$ \\
\hline GYP (g) & $\begin{array}{l}7.49 \pm 2.35 \\
n=62\end{array}$ & $\begin{array}{l}5.51 \pm 1.28 \\
p<0.0001, n=42\end{array}$ & $\begin{array}{l}9.64 \pm 2.39 \\
p=0.004, n=20\end{array}$ & $\begin{array}{l}8.9 \pm 2.58 \\
p=0.0250, n=22\end{array}$ \\
\hline GY (ton) & $\begin{array}{l}3.96 \pm 0.12 \\
n=4\end{array}$ & $\begin{array}{l}3.52 \pm 0.09 \\
p=0.0011, n=4\end{array}$ & $\begin{array}{l}5.26 \pm 0.36 \\
n=2\end{array}$ & $\begin{array}{l}4.57 \pm 0.03 \\
n=2\end{array}$ \\
\hline
\end{tabular}

(Supplementary Fig. 4). ABA synthesis pathway genes, however, were upregulated, including phytoene synthase (PSY, a committed step to flux GGPP into carotenoid synthesis), violaxanthin deepoxidase (VDE), and 9-cis-epoxycarotenoid dioxygenase (NCED, a crucial step for ABA synthesis from carotenoid) (Fig. 5b). qRTPCR experiments further confirmed the up-regulation of these genes in $\operatorname{tg} w 1$ (Fig. 5c-e).

The ABA content showed a significant increase in the tgw1 mutant (Fig. 5f). On the contrary, the KAT-2B transgenic lines accumulated less ABA (Fig. 5g), suggesting a negative correlation between the biological function of KAT-2B (JA content) and ABA biosynthesis in wheat. To be consistent with the ABA content in the tgw1 mutant, a wheat leaf senescence-associated marker gene, $S A G 3^{29}$, increased in a qRT-PCR assay (Fig. 5h). Exogenous ABA treatment enhanced the expression of $S A G 3$ in young seedlings (Fig. 5i).

The above data suggested that the JA synthesis defect heightened the expression of ABA synthesis genes and increased ABA, which enhanced the expression of SAG3. Early senescence marked by $S A G 3$ increase could be a potential link between the increased $\mathrm{ABA}$ and reduced chlorophyll contents in the tgw1 mutant, as the other regulation, besides leaf area, on the source limitation for grains (Fig. 5j).

\section{Discussion}

Through this work, we discovered a unique role of wheat $K A T-2 B$ in regulating GW through directly controlling de novo JA synthesis. However, the mutational effects of the wheat KAT-2B gene are pleiotropic, e.g., negatively affecting JA content and leaf and pollen development, and positively increasing ABA content. Therefore, the mechanism underlying the KAT- $2 B$ function is likely very complicated.

In our study, wheat $\operatorname{tg} w 1$ led to expression changes of multiple essential synthesis genes in several plant hormones, e.g., JA, ABA, and GA (Table 2). In metabolite analyses, JA showed an apparent decrease, whereas no significant change could be detected in the contents of IAA and total free lipids between $\operatorname{tg} w 1$ and the control samples analyzed. $\beta$-oxidation was reported to catalyze the conversion of IBA to IAA, which affects root hair elongation and cotyledon $\operatorname{size}^{30}$. In the plant, however, IAA was mainly synthesized from 1-tryptophan via indole-3-pyruvate by the TAA and YUC families in Arabidopsis ${ }^{31}$. Our data here agreed with the latter report that $\beta$-oxidation catalyzed by KAT-2B might not play a significant role in IAA synthesis in wheat.

Oxidized lipids, including JA, are reported at low levels in plants in earlier studies. In Arabidopsis, oxidized lipids composition was changed induced upon treatment at $4{ }^{\circ} \mathrm{C}$ or pathogen inoculation, but the highest amount upon treatment $(\sim 4.3 \mathrm{nmol}$ per $\mathrm{mg}$ of dry mass) was still much lower than those of normal-chain phospholipids and galactolipids ( $259 \mathrm{nmol}$ per mg of dry mass $)^{32}$. In wheat, oxidized lipids were reported to increase upon high-temperature treatment in the leaves of a susceptible cultivar ${ }^{33}$. However, the contents of ox-lipids ranged from 0.0005 to 0.015 mass spectral signal per mg dry mass, which was relatively low compared to the total lipids $(50-200)^{33}$. Therefore, the change in JA content could only have a minor effect on the total content of lipids, which was an under-detectable level in our system. 
a

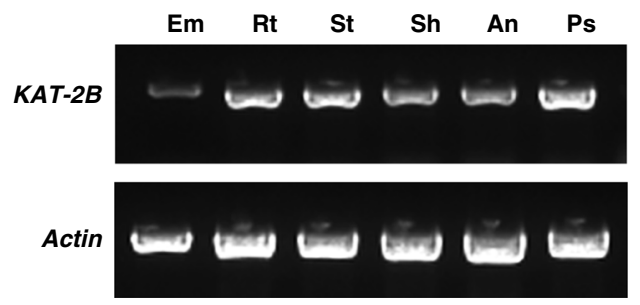

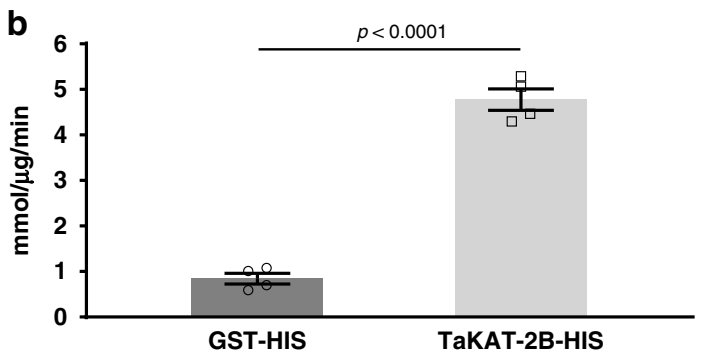

C
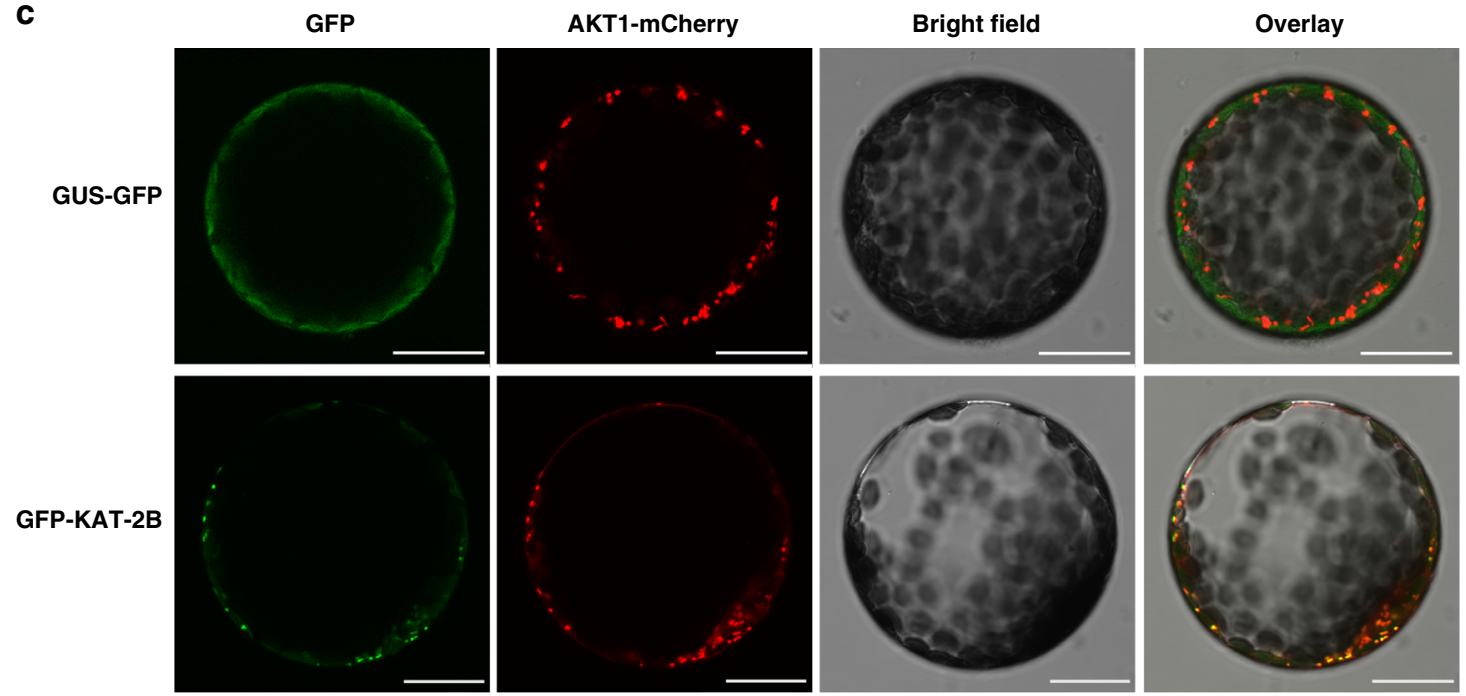

d
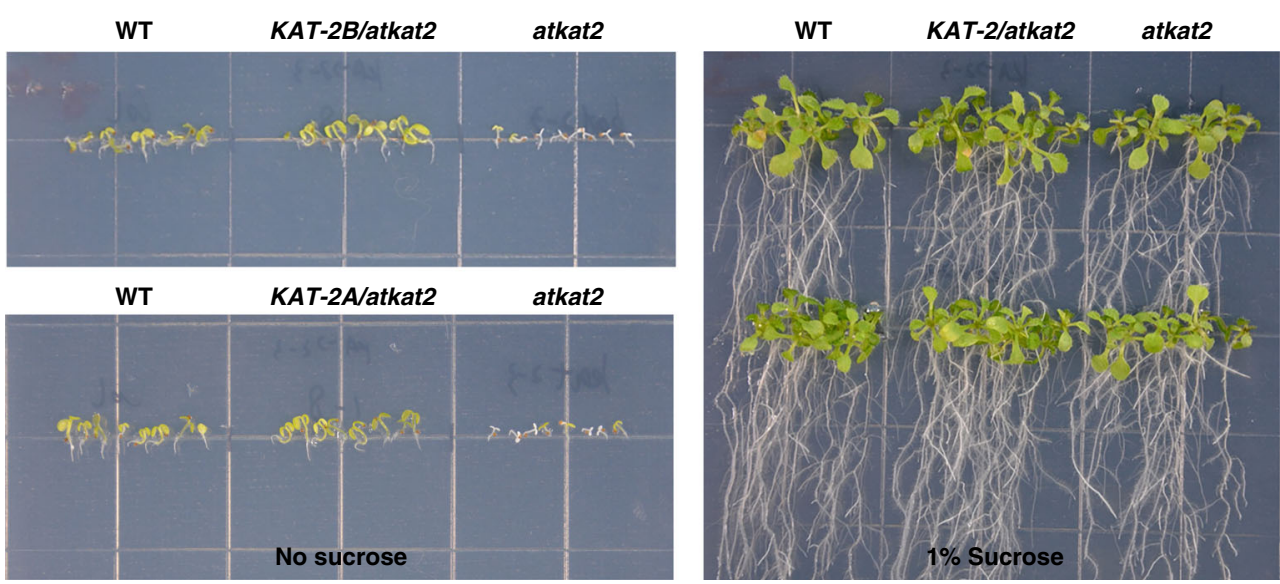

Fig. 4 KAT-2B is an active thiolase in the peroxisome. a RT-PCR analysis of $K A T-2 B$ in six tissues of WT. b Specific biochemical activity of recombinant KAT-2B. $n=4$. Data are represented as mean \pm SEM, and $p$ values are indicated by two-tailed unpaired $t$ test. c Subcellular localization of GFP-KAT-2B fusion protein in protoplast. Images are representative of three independent experiments. GUS-GFP stands for a soluble protein. AKT1-mCherry is a peroxisome marker. Scale bar $=25 \mu \mathrm{m}$. d Rescue of the Arabidopsis kat2 mutant in a sucrose-free medium by wheat KAT-2 transgene. Note that Arabidopsis kat2 mutant is lethal in a sucrose-free medium. Each grid represents $1 \mathrm{~cm}^{2}$ on the plate. Source data underlying Fig. $4 a-c$ are provided as a Source Data file.

In most plants, phytohormones are essential regulators of plant leaf senescence, and many of these altered senescence phenotypes occur as a result of altered hormone signaling ${ }^{34}$. In Arabidopsis, a JA-inducible gene, Dof2.1 (DNA binding-withone-finger), acted as an enhancer of JA-induced leaf senescence through the MYC2-Dof2.1-MYC2 feed-forward transcriptional loop. In addition to the decrease of JA, the up-regulation of $\mathrm{ABA}$ could also be important for the grain defect in tgwl. The Arabidopsis kat2 mutant showed an increase in $\mathrm{ABA}$ response ${ }^{35}$. In rice, an NAM/ATAF $1 / 2 / \mathrm{CUC} 2$ (NAC) transcription factor ONAC054 bound the promoter region and played a crucial role in the regulation of $\mathrm{ABA}$ signaling and senescence-related genes, e.g., OsABI5 and $N Y C 1^{36}$. A MYB-related transcription factor Oryza sativa RADIALIS-LIKE3 (OsRL3), induced by ABA, promoted the expression levels of chlorophyll-degradation and senescence-associated genes ${ }^{37}$. In maize, $\mathrm{ABA}$, at high concentration, increased sterility and abortion ${ }^{38,39}$. Regulation of kernel weight by ABA was detected, which was accompanied by an inhibitory effect at high concentration to sucrose synthase and starch synthase activities ${ }^{40}$. In this study, a wheat leaf senescence marker gene, $S A G 3^{29}$, was upregulated by exogenous $\mathrm{ABA}$ treatment and the over-accumulated endogenous $\mathrm{ABA}$ in the tgw1 mutant. Hence, the ABA content change could explain the decrease of chlorophyll contents in the tgw1 mutant leaves, a 
a

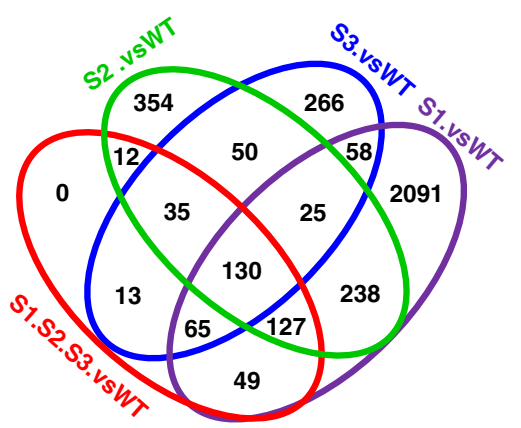

C

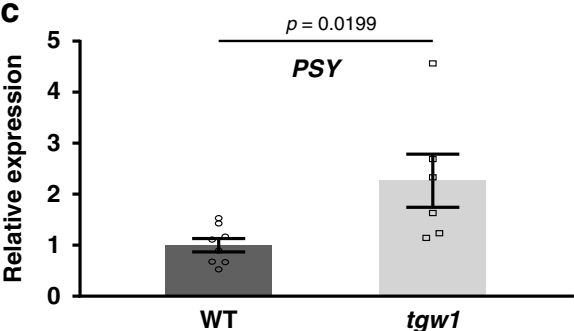

b
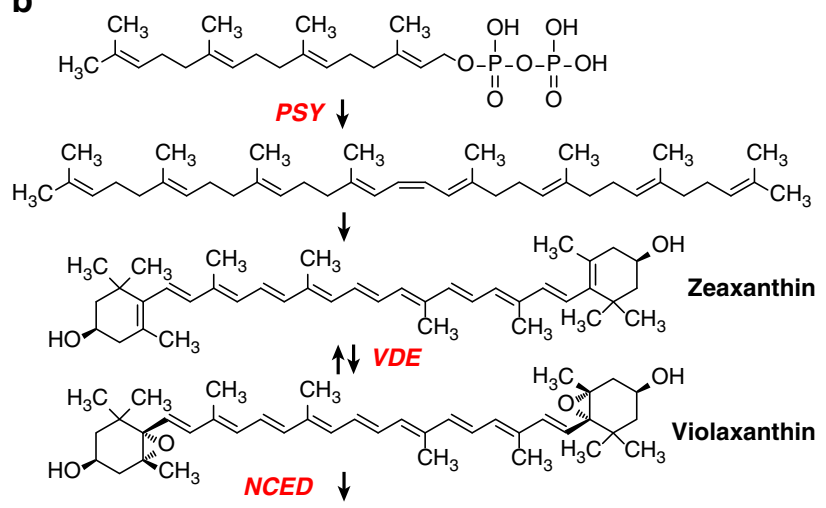

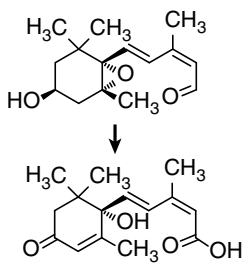

Xanthoxin

Abscisic acid

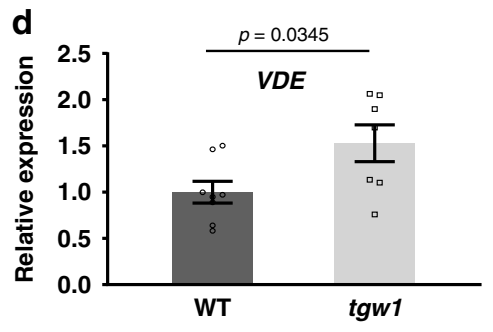

g

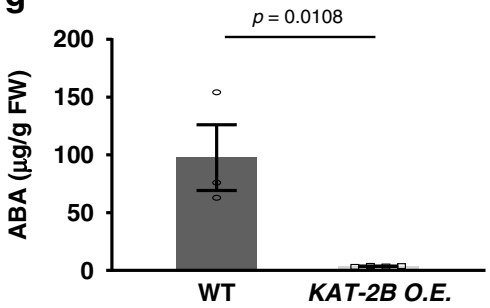

j

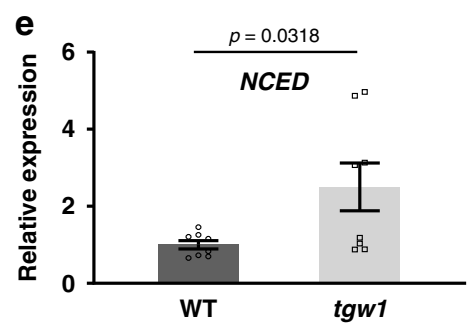

h

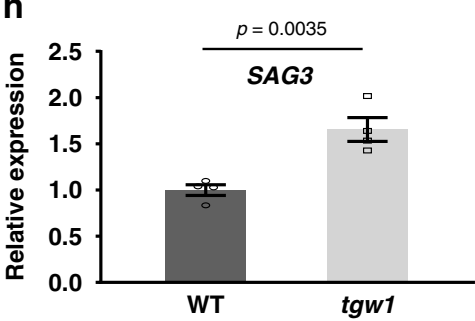

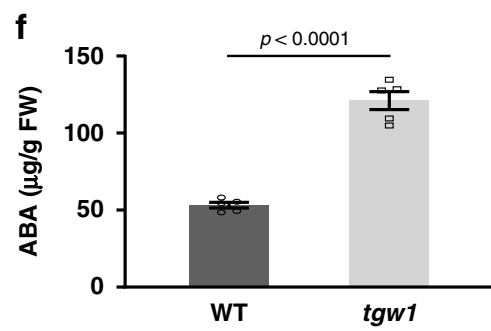

i

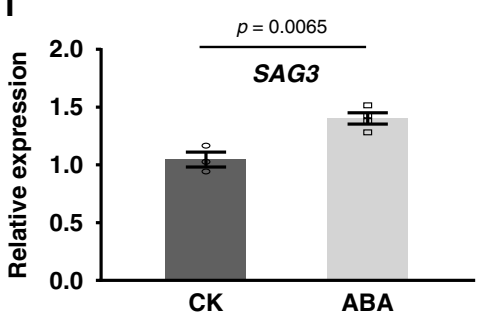

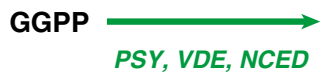

ABA

GGPP
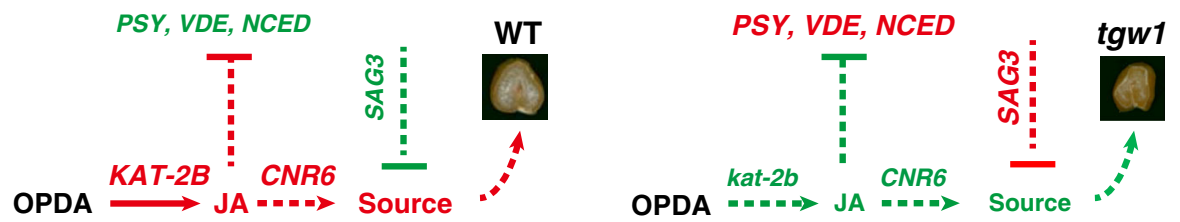

ABA

OPDA $=\cdots \cdots$ JA $=\cdots$ Source

Fig. 5 Regulation of ABA synthesis in tgw1. a Venn diagram representation of differential expression genes between WT and the tgw1 backcrossed mutant. b Schematic representation of the ABA synthesis pathway. PSY, VDE, and NCED were upregulated in tgw1 mutant. c-e Relative expression level of PSY, VDE, and NCED in WT and tgw1 mutant from at least four independent biological samples. $\mathbf{f}$ Quantification of ABA in WT and tgw1 mutant. $n=5$. $\mathbf{g}$ Quantification of ABA in WT $(n=3)$ and transgenic plants $(n=4)$. $\mathbf{h}$ Relative expression level of senescence-associated gene 3 (SAG3) in WT and tgw1 mutant. $n=4$. i Expression change of SAG3 upon $80 \mu \mathrm{M} \mathrm{ABA}$ treatment. $n=4$. j A predicted working model for GW regulation in WT or tgw 1 mutant. Red, upregulated. Green, downregulated. WT and tgw1 grains were cross-dissected to show grain width. Data in c-i are represented as mean \pm SEM, and $p$ values are indicated by two-tailed unpaired $t$ test. Source data underlying Fig. $5 a$ and $c-i$ are provided as a Source Data file.

crucial component to determine the photosynthesis rate in the source tissue.

In addition, leaf area change could be another negative player for the source energy capture in the tgw 1 mutant. In tgw 1 mutant leaves, the expression of JA-inducible CNR6 declined, possibly owing to the deficient in JA content. In previous reports, CNR1 played a negative role in the regulation of organ size and crop yield ${ }^{41}$. In wheat, however, it is very likely that CNR6 could be a positive regulator on the cell number, which could be tested if necessary genetic materials were available. The reduced cell number and final leaf size played a negative effect on the source for grain filling. Source limitation to GW was noted in some cultivars under different environments ${ }^{26}$. Source for grain filling was found to be a limiting factor when wheat sink capacity increased as exampled in bread wheat recombinant 
Table 2 List of differential genes involved in plant hormone metabolism between tgw1 and WT.

\begin{tabular}{|c|c|c|}
\hline Gene-ID & $\begin{array}{l}\log _{2} \text { (fold } \\
\text { change) }\end{array}$ & Annotation \\
\hline \multicolumn{3}{|l|}{$J A$} \\
\hline TraesCS2A02G525500.1 & -1.39 & Lipoxygenase \\
\hline TraesCS2B02G555400.1 & -1.49 & Lipoxygenase \\
\hline TraesCS4A02G061800.1 & -1.49 & Allene oxide synthase \\
\hline TraesCS4A02G061900.1 & -1.13 & Allene oxide synthase \\
\hline TraesCS4B02G037700.2 & -1.11 & Lipoxygenase \\
\hline TraesCS4B02G037900.1 & -1.67 & Lipoxygenase \\
\hline TraesCS4B02G237600.1 & -1.21 & Allene oxide synthase \\
\hline TraesCS5A02G007900.1 & -1.28 & Lipoxygenase \\
\hline TraesCS5B02G006500.1 & -1.24 & Lipoxygenase \\
\hline TraesCS6B02G160400.1 & -1.64 & Lipoxygenase \\
\hline TraesCS6B02G432600.1 & -4.07 & $\begin{array}{l}\text { 3-ketoacyl-CoA } \\
\text { thiolase-like }\end{array}$ \\
\hline TraesCS6B02G056800.2 & -1.18 & Catalase \\
\hline TraesCS6A02G041700.1 & -1.23 & Catalase \\
\hline TraesCS1A02G030400.1 & -2.00 & $\begin{array}{l}\text { Jasmonate O- } \\
\text { methyltransferase }\end{array}$ \\
\hline \multicolumn{3}{|l|}{$A B A$} \\
\hline TraesCS5A02G020900.1 & 1.07 & $\begin{array}{l}\text { Phytoene synthase, } \\
\text { chloroplastic-like }\end{array}$ \\
\hline TraesCS2B02G297800.1 & 1.24 & $\begin{array}{l}\text { Violaxanthin de- } \\
\text { epoxidase }\end{array}$ \\
\hline TraesCS5A02G374000.1 & 1.92 & $\begin{array}{l}\text { 9-cis-epoxycarotenoid } \\
\text { dioxygenase }\end{array}$ \\
\hline \multicolumn{3}{|r|}{ se } \\
\hline TraesCS2A02G425500.1 & -1.16 & Kaurene synthase \\
\hline
\end{tabular}

inbred lines with contrasting $\mathrm{GN}^{42}$. Therefore, JA defect followed by the accumulation of $\mathrm{ABA}$, a decrease of leaf development, and other unknown factors could all be involved in the GW regulation in tgw1.

Following the experimental design on a breeding selection of wheat kernel weight ${ }^{43}$, our field trials showed that KAT-2B transgenic lines could significantly increase yield. However, it is noted that the plot size we used (one $1 \mathrm{~m}$ row or $1 \mathrm{~m}^{2}$ in the 2019 and 2020 experiments, respectively) was smaller than what breeders usually use in breeding selection. In addition, our field trials were conducted in the same location for two years. To validate the breeding applicability of the $K A T-2 B$ gene, largerscale field trails across multiple sites with different soil types and environmental conditions are still needed. Furthermore, we used WT rather than null segregant as control. We should point out that null segregant is more appropriate for this purpose.

In conclusion, our work establishes that wheat $K A T-2 B$ positively regulates $\mathrm{GW}$, a vital component of wheat yield. Consequently, this gene is useful for further dissecting the molecular basis, especially the role of JA, underlying wheat grain development. Because we found that overexpression of $K A T-2 B$ substantially increased GW and grain yield per plant, the potential value of this gene in developing wheat cultivars with improved GW is worthy of investigation. The use of precise genome engineering techniques, e.g., CRISPR-Cas9 $9^{44}$, may facilitate the modification of $K A T-2 B$ function and JA biosynthesis during wheat grain development. Thus, our study offers new clues and possibilities for studying wheat grain development.

\section{Methods}

Plant materials and growth conditions. All the mutants and transgenic wheat used in this work were in Triticum turgidum (tetraploid, AABB genome) Kronos ecotype background. Wild-type seeds were treated with $0.7 \%$ EMS for $16 \mathrm{~h}$ at $25^{\circ} \mathrm{C}$ to generate an EMS mutant library. $\mathrm{M}_{2}$ generation siblings were phenotyped to select mutants with precise changes. $\mathrm{BC}_{1} \mathrm{~F}_{2}$ generation plants were used for segregation tests, and homozygous plants were selected for subsequent step analyses. Arabidopsis kat2 mutant was ordered from Arabidopsis Research Center (ABRC). All plants were cultivated in a growth chamber at $22{ }^{\circ} \mathrm{C}, 65 \%$ relative humidity, and 16-hour photoperiod. At least two generations of transgenic and EMS mutants were observed with consistent phenotype.

The materials for phenotypic evaluation were grown at the Fudan University Experimental Station $\left(31^{\circ} 27^{\prime} \mathrm{N}, 121^{\circ} 52^{\prime} \mathrm{E}\right)$ in Shanghai, representing the southern part of the Yangtze Spring Wheat Zone. For the 2019 experiment, the three genotypes (WT, $\operatorname{tg} w 1, K A T-2 B$ O.E.) were sown in a single row with $1 \mathrm{~m}$ in length spaced $0.25 \mathrm{~m}$ apart with 25 plants per row in December 2018, and plants were harvested in June 2019. For the 2020 experiment, each plot consisted of four 1meter row spaced $0.25 \mathrm{~m}$ apart with 25 plants per row and 100 seeds were sown in each plot in December 2019. The four genotypes (WT, $\operatorname{tg} w 1, K A T-2 B$ O.E. $3^{\#}$, and $\left.K A T-2 B O . E .4^{\#}\right)$ were arranged using a $4 \times 4$ lattice design according to a previous publication ${ }^{43}$. Before sowing, compound fertilizer (N: P: $\mathrm{K}=25 \%$ : $15 \%$ : $8 \%$, Hongsifang, Anhui, China) was applied at $191 \mathrm{~kg} \mathrm{ha}^{-1}$. Field management followed local agricultural practices. In the following June, randomly sampled plants were used to measure tiller number at harvest, total spikelet number per spike, spike length $(\mathrm{cm})$, GNS, GWS, TKW and grain yield per plant. Kernels in the remaining plants were harvested and dried in an oven at $40{ }^{\circ} \mathrm{C}$ for a week to a constant weight. Grain yield per square meter was obtained by summarizing the GWs in the plot, and converted to ton per ha for the 2020 experiment.

BSA to clone TGW1. RNA was extracted from mature leaves of 2-month $\mathrm{BC}_{1} \mathrm{~F}_{2}$ generation plants using TRIzol Reagent according to the user manual. We next mixed RNA samples from eight individual homozygous plants together at an equal ratio to form one group. We prepared three groups of WT and homozygous mutants, respectively, to construct PE300 libraries and sequenced in Illumina Hiseq4000 (Shanghai Hanyu Bio-Tech). Around 10 Giga high-quality data were collected from each group and analyzed to call homozygous single-nucleotide polymorphisms (SNPs) with at least five reads in any group using the software BCF Tools v0.1.19. Differentially expressed genes were filtered following the rule of Fold change $\mid>2$, FDR ( $q$ value) $<0.001$, and RPKM over 20 in at least one sample using an MA-plot-based method with Random sampling model in DEGseq v1.20.0 ${ }^{45}$. Gene assembly and annotation were conducted according to wheat assembly (TGACv1.1).

Subcellular localization and transgenic plants. $K A T-2 B \mathrm{cDNA}$ was cloned from WT plants, ligated into pDNOR207 by BP reaction (Invitrogen, Life Technologies, Carlsbad, CA, USA), and confirmed by sequencing. For subcellular localization analysis, KAT-2B was incorporated into pMDC43 (Pro ${ }_{355}$ : GFP-KAT-2B) by LR reaction (Invitrogen), transformed into Agrobacterium GV3101, infiltrated into tobacco leaves, and observed with an inverted confocal microscope equipped with a $\times 40$ oil-corrected objective in Leica TCS SP8 (Leica, Microsystems, Mannheim, Germany) at $48 \mathrm{~h}^{46}$. To complement the Arabidopsis kat2 mutant, KAT-2 was incorporated into a gateway transgenic vector under the drive of maize ubiquitin

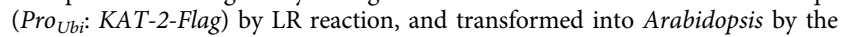
flower dip method using Agrobacterium GV3101. Immature embryos were collected and inoculated with Agrobacterium to generate transgenic wheat according to earlier reports ${ }^{47}$.

Gene expression and protein accumulation analysis. For gene expression pattern analyses, embryos were collected from germinating seeds. Roots were collected from the 1-week seedlings. Stems, shoots, anthers, and pistils were collected from flowering plants. Seven-day-old wheat seedlings were used for various treatments. Seedling leaves were sprayed with $10 \mu \mathrm{M}$ MeJA, $200 \mu \mathrm{M}$ DIEC, or $80 \mu \mathrm{M}$ ABA. Seedings without any treatment were used as controls. RNA was extracted from seedling leaves using TRIzol according to the manufacture's user manual (Invitrogen). cDNAs were synthesized using the oligo (dT) primer (TAKARA BIO INC, Tokyo, Japan). Quantitative real-time RT-PCR was conducted using SYBR Green Master (TAKARA BIO INC) with gene-specific primers. Primer sequences are provided in Supplementary Table 2.

Total proteins were extracted from leaves of transgenic or WT and analyzed in a Western blot experiment with an anti-KAT-2 antibody generated for this study in a local company (Abmart, Shanghai, China) at 1:400 dilution. A goat anti-mouse IgG HRP (M21001, Abmart) was used to recognize the primary antibody at 1:8000 dilution according to the manufacture user manual. The samples were developed with a basic ECL kit (AB Clonal) in Tanon 5200 Multi imaging system (Tanon Science \& Technology Co., Ltd., Shanghai, China). Total protein was extracted from fully expanded leaves in a growth chamber and analyzed as described to check the protein level of complementation lines.

Recombinant protein purification and activity analyses. Recombinant KAT-2B proteins were purified from Rosetta ${ }^{48}$. KAT- $2 B$ was incorporated into a gateway expression vector $\left(\mathrm{P}_{\mathrm{T} 7}:\right.$ His9-AttR: $\left.\mathrm{T}_{\mathrm{T} 7}\right)$ by $\mathrm{LR}$ reaction, and transformed into $E$. Coli, Rosetta, by a heat shock method. For protein induction, $0.5 \mathrm{mM}$ IPTG was added into cell cultures at $\mathrm{OD}_{600} \sim 0.8$ in a shaker set at $18^{\circ} \mathrm{C}$. Cells were collected 
from overnight cultures, sonicated to lysis and purified by Ni-NTA beads according to the user manual ${ }^{49}$. For activity assay, $2 \mu \mathrm{g}$ recombinant KAT-2B proteins were mixed with a reaction buffer with $100 \mu \mathrm{M} \mathrm{CoA}$ and $50 \mu \mathrm{M}$ acetoacetyl CoA at $25^{\circ} \mathrm{C}$. UV absorbance at $232 \mathrm{~nm}$ was read every $0.5 \mathrm{~min}$ for $5 \mathrm{~min} .100 \mathrm{mM}$ monovalent cations $\left(\mathrm{K}^{+}\right.$and $\mathrm{Na}^{+}$) or $50 \mathrm{mM}$ divalent cations (e.g., $\mathrm{Mg}^{2+}$ ) were added in the reaction buffer to quantify absorbance changes to study the effects of ions on KAT-2B activity. $100 \mathrm{mM}$ phosphate buffers at $\mathrm{pH} 6,6.5,7.0,7.5,8.0,8.5$, and 9 were used with $100 \mathrm{mM} \mathrm{NaCl}$ to quantify absorbance changes at $25^{\circ} \mathrm{C}$ to study the effects of $\mathrm{pH}$ on KAT-2B activity.

Metabolite profiling analysis. Leaves were collected from 1-week-old seedlings and embryos from mature grains. The samples were ground into a fine powder, extracted with $70 \%$ ethanol plus $50 \mu \mathrm{L}$ of internal standard, and analyzed by LC/ $\mathrm{MS}^{50}$. Quantification of hormones was calculated by comparing with internal isotope standards (JA: $\mathrm{C}_{12} \mathrm{H}_{13} \mathrm{D}_{5} \mathrm{O}_{3}$; IAA: $\mathrm{C}_{10} \mathrm{H}_{7} \mathrm{D}_{2} \mathrm{NO}_{2} ; \mathrm{ABA}: \mathrm{C}_{15} \mathrm{H}_{14} \mathrm{D}_{6} \mathrm{O}_{4}$ ) dissolved in methanol at $200 \mathrm{ppb}^{51}$ to quantify the contents of plant hormones. For the quantification of endogenous JA content, 2-week old seedling leaves were homogenized in ice-cold phosphate-buffered saline ( $\mathrm{pH}$ 7.4). After centrifugation at $3000 \times g$ for $20 \mathrm{~min}$, the supernatant was assayed by an ELISA kit (Zhen Ke Biological Technology Co., Ltd., Shanghai, China).

Oxidized lipid analysis was performed according to previous literature ${ }^{52}$. In brief, $2 \mathrm{gm}$ of the fully expanded leaf was collected in the field and ground into fine powder in liquid nitrogen in a high-though put homogenizer. In all, $2 \mathrm{~mL}$ of cold methanol was added immediately into the sample to kill enzymes. In all, $10 \mu \mathrm{L}$ of internal standard ( $2 \mathrm{mg}$ heptadecanoic acid methyl ester $/ \mathrm{mL}$ hexane) and $50 \mu \mathrm{L}$ of $1 \mu \mathrm{M}$ acetic acid was added and mixed well. After centrifugation at $21,000 \times g$ for $15 \mathrm{~min}$, the supernatant was removed into a new $10 \mathrm{~mL}$ glass tube. Then $1 \mathrm{~mL}$ of hexane: ethyl acetate $\left(1: 1\right.$, v:v) was added and shaken in a $37^{\circ} \mathrm{C}$ shaker for 1 hour. The supernatant was removed into the $10 \mathrm{~mL}$ tube. After wash three times with hexane: ethyl acetate, $2 \mathrm{~mL}$ of $\mathrm{ddH}_{2} \mathrm{O}$ was added and mixed well in the $10 \mathrm{~mL}$ glass tube. The solvent phase was removed into a new tube and dried under a flow of nitrogen gas. The sample was dissolved in $0.5 \mathrm{~mL}$ of chloroform: isopropanol $(2: 1$, $\mathrm{v}: \mathrm{v})$ and loaded into an $\mathrm{LC}-\mathrm{NH}_{2}$ column. The column was washed twice with chloroform: isopropanol and eluted with ethyl acetate: acetic acid (2:1, v:v). The sample was dried and dissolved in $1 \mathrm{~mL}$ of methanol: hexane (4:1, v:v). The sample was cooled in liquid nitrogen for $15 \mathrm{~min}$, mixed with $0.1 \mathrm{~mL}$ of acetyl chloride, and kept in the dark overnight. The reaction was stopped with $2.5 \mathrm{~mL}$ of $6 \% \mathrm{~K}_{2} \mathrm{CO}_{3}$ following extraction with hexane. The sample was dried and analyzed by GC/MS. Oxidized lipid characterization was performed based on the mass spectrum, according to a literature ${ }^{52}$

Reporting summary. Further information on research design is available in the Nature Research Reporting Summary linked to this article.

\section{Data availability}

Data supporting the findings of this work are available within the paper and its Supplementary Information files. A reporting summary for this Article is available as a Supplementary Information file. The data sets and plant materials generated and analyzed during the current study are available from the corresponding author upon request. RNA-seq data have been uploaded to NCBI with accession number PRJNA673987 or wheat expression database in the Triticeae Multi-omics Center [http:// 202.194.139.32/]. Source data are provided with this paper.

Received: 31 October 2018; Accepted: 15 November 2020; Published online: 08 December 2020

\section{References}

1. Quintero, A., Molero, G., Reynolds, M. P. \& Calderini, D. F. Trade-off between grain weight and grain number in wheat depends on GxE interaction: a case study of an elite CIMMYT panel (CIMCOG). Eur. J. Agron. 92, 17-29 (2018).

2. Acreche, M. \& Slafer, G. Grain weight response to increases in number of grains in wheat in a Mediterranean area. Field Crops Res. 98, 8 (2006).

3. Abbo, S. et al. Plant domestication versus crop evolution: a conceptual framework for cereals and grain legumes. Trends Plant Sci. 19, 351-360 (2014).

4. Li, W. \& Yang, B. Translational genomics of grain size regulation in wheat. Theor. Appl. Genet. 130, 1765-1771 (2017).

5. Peng, J. et al. Domestication quantitative trait loci in Triticum dicoccoides, the progenitor of wheat. Proc. Natl. Acad. Sci. USA 100, 2489-2494 (2003).

6. Zuo, J. \& Li, J. Molecular genetic dissection of quantitative trait loci regulating rice grain size. Аnnu. Rev. Genet. 48, 99-118 (2014).

7. Ashikari, M. et al. Cytokinin oxidase regulates rice grain production. Science 309, 741-745 (2005)
8. Guo, T. et al. GRAIN SIZE AND NUMBER1 negatively regulates the OsMKKK10-OsMKK4-OsMPK6 cascade to coordinate the trade-off between grain number per panicle and grain size in rice. Plant Cell 30, 871-888 (2018).

9. Ishimaru, K. et al. Loss of function of the IAA-glucose hydrolase gene TGW6 enhances rice grain weight and increases yield. Nat. Genet .45, 707-711 (2013).

10. Li, Y. et al. Natural variation in GS5 plays an important role in regulating grain size and yield in rice. Nat. Genet. 43, 1266-1269 (2011).

11. Liu, L. et al. Activation of Big Grain1 significantly improves grain size by regulating auxin transport in rice. Proc. Natl. Acad. Sci. USA 112, 11102-11107 (2015).

12. Song, X. J., Huang, W., Shi, M., Zhu, M. Z. \& Lin, H. X. A. QTL for rice grain width and weight encodes a previously unknown RING-type E3 ubiquitin ligase. Nat. Genet. 39, 623-630 (2007).

13. Wang, S. et al. Control of grain size, shape and quality by OsSPL16 in rice. Nat. Genet. 44, 950-954 (2012).

14. Wang, Y. et al. Simultaneous editing of three homoeoalleles in hexaploid bread wheat confers heritable resistance to powdery mildew. Nat. Biotechnol. 32, 947-951 (2014)

15. Wang, Y. et al. Copy number variation at the GL7 locus contributes to grain size diversity in rice. Nat. Genet. 47, 944-948 (2015).

16. Zhang, X. et al. Rare allele of OsPPKL1 associated with grain length causes extra-large grain and a significant yield increase in rice. Proc. Natl. Acad. Sci. USA 109, 21534-21539 (2012).

17. Lyons, R., Manners, J. M. \& Kazan, K. Jasmonate biosynthesis and signaling in monocots: a comparative overview. Plant Cell Rep. 32, 815-827 (2013).

18. Wasternack, C., Forner, S., Strnad, M. \& Hause, B. Jasmonates in flower and seed development. Biochimie 95, 79-85 (2013).

19. Huo, X. et al. NOG1 increases grain production in rice. Nat. Commun. 8, 1497 (2017).

20. Cai, Q. et al. Jasmonic acid regulates spikelet development in rice. Nat. Commun. 5, 3476 (2014).

21. Zhao, Y. et al. A wheat allene oxide cyclase gene enhances salinity tolerance via jasmonate signaling. Plant Physiol. 164, 1068-1076 (2014).

22. Eltayeb Habora, M. E., Eltayeb, A. E., Oka, M., Tsujimoto, H. \& Tanaka, K. Cloning of allene oxide cyclase gene from Leymus mollis and analysis of its expression in wheat-Leymus chromosome addition lines. Breed. Sci. 63, 68-76 (2013).

23. Sagar, M., Pandey, N., Qamar, N., Singh, B. \& Shukla, A. Domain analysis of 3 Keto Acyl-CoA synthase for structural variations in Vitis vinifera and Oryza brachyantha using comparative modelling. Interdiscip. Sci. 7, 7-20 (2015).

24. Wiszniewski, A. A., Bussell, J. D., Long, R. L. \& Smith, S. M. Knockout of the two evolutionarily conserved peroxisomal 3-ketoacyl-CoA thiolases in Arabidopsis recapitulates the abnormal inflorescence meristem 1 phenotype. J. Exp. Bot. 65, 6723-6733 (2014)

25. Footitt, S., Cornah, J. E., Pracharoenwattana, I., Bryce, J. H. \& Smith, S. M. The Arabidopsis 3-ketoacyl-CoA thiolase-2 (kat2-1) mutant exhibits increased flowering but reduced reproductive success. J. Exp. Bot. 58, 2959-2968 (2007).

26. Fischer, R. A. \& HilleRisLamber, D. Effect of environment and cultivar on source limitation to grain weight in wheat. Aust. J. Agric. Res. 29, 16 (1978).

27. Nelson, B. K., Cai, X. \& Nebenfuhr, A. A multicolored set of in vivo organelle markers for co-localization studies in Arabidopsis and other plants. Plant $J$. 51, 1126-1136 (2007)

28. Germain, V. et al. Requirement for 3-ketoacyl-CoA thiolase-2 in peroxisome development, fatty acid beta-oxidation and breakdown of triacylglycerol in lipid bodies of Arabidopsis seedlings. Plant J. 28, 1-12 (2001).

29. Kajimura, T., Mizuno, N. \& Takumi, S. Utility of leaf senescence-associated gene homologs as developmental markers in common wheat. Plant Physiol. Biochem. 48, 851-859 (2010).

30. Strader, L. C., Culler, A. H., Cohen, J. D. \& Bartel, B. Conversion of endogenous indole-3-butyric acid to indole-3-acetic acid drives cell expansion in Arabidopsis seedlings. Plant Physiol. 153, 1577-1586 (2010).

31. Kasahara, H. Current aspects of auxin biosynthesis in plants. Biosci. Biotechnol. Biochem. 80, 34-42 (2016).

32. $\mathrm{Vu}, \mathrm{H}$. S. et al. Direct infusion mass spectrometry of oxylipin-containing Arabidopsis membrane lipids reveals varied patterns in different stress responses. Plant Physiol. 158, 324-339 (2012).

33. Narayanan, S., Prasad, P. V. \& Welti, R. Wheat leaf lipids during heat stress: II. Lipids experiencing coordinated metabolism are detected by analysis of lipid co-occurrence. Plant Cell Environ. 39, 608-617 (2016).

34. Li, Z., Peng, J., Wen, X. \& Guo, H. Gene network analysis and functional studies of senescence-associated genes reveal novel regulators of Arabidopsis leaf senescence. J. Integr. Plant Biol. 54, 526-539 (2012).

35. Jiang, T., Zhang, X. F., Wang, X. F. \& Zhang, D. P. Arabidopsis 3-ketoacylCoA thiolase-2 (KAT2), an enzyme of fatty acid beta-oxidation, is involved in ABA signal transduction. Plant Cell Physiol. 52, 528-538 (2011). 
36. Sakuraba, Y. et al. Multilayered regulation of membrane-bound ONAC054 is essential for abscisic acid-induced leaf senescence in rice. Plant Cell 32, 630-649 (2020).

37. Park, D.-Y. et al. The MYB-related transcription factor RADIALIS-LIKE3 (OsRL3) functions in ABA-induced leaf senescence and salt sensitivity in rice. Environ. Exp. Bot. 156, 86-95 (2018)

38. Kakumanu, A. et al. Effects of drought on gene expression in maize reproductive and leaf meristem tissue revealed by RNA-Seq. Plant Physiol. 160, 846-867 (2012).

39. Wang, Z., Mambelli, S. \& Setter, T. L. Abscisic acid catabolism in maize kernels in response to water deficit at early endosperm development. Ann. Bot. 90, 623-630 (2002).

40. Zhang, L. et al. Regulation of maize kernel weight and carbohydrate metabolism by abscisic acid applied at the early and middle post-pollination stages in vitro. J. Plant Physiol. 216, 1-10 (2017).

41. Guo, M. et al. Cell Number Regulator1 affects plant and organ size in maize: implications for crop yield enhancement and heterosis. Plant Cell 22, 1057-1073 (2010).

42. Alonso, M. P. et al. Analysis of sink/source relations in bread wheat recombinant inbred lines and commercial cultivars under a high yield potential environment. Eur. J. Agron. 93, 82-87 (2018).

43. $\mathrm{Li}, \mathrm{T}$. et al. Variation in allelic frequencies at loci associated with kernel weight and their effects on kernel weight-related traits in winter wheat. Crop J. 7, 30-37 (2019).

44. Zong, Y. et al. Precise base editing in rice, wheat and maize with a Cas9cytidine deaminase fusion. Nat. Biotechnol. 35, 438-440 (2017).

45. Wang, L., Feng, Z., Wang, X. \& Zhang, X. DEGseq: an R package for identifying differentially expressed genes from RNA-seq data. Bioinformatics 26, 136-138 (2010).

46. Curtis, M. D. \& Grossniklaus, U. A gateway cloning vector set for highthroughput functional analysis of genes in planta. Plant Physiol. 133, 462-469 (2003).

47. Gou, J. Y. et al. Wheat stripe rust resistance protein WKS1 reduces the ability of the thylakoid-associated ascorbate peroxidase to detoxify reactive oxygen species. Plant Cell 27, 1755-1770 (2015).

48. Gou, J. Y., Yu, X. H. \& Liu, C. J. A hydroxycinnamoyltransferase responsible for synthesizing suberin aromatics in Arabidopsis. Proc. Natl. Acad. Sci. USA 106, 18855-18860 (2009).

49. Gou, J. Y. et al. Acetylesterase-mediated deacetylation of pectin impairs cell elongation, pollen germination, and plant reproduction. Plant Cell 24, 50-65 (2012).

50. Gou, J. Y., Wang, L. J., Chen, S. P., Hu, W. L. \& Chen, X. Y. Gene expression and metabolite profiles of cotton fiber during cell elongation and secondary cell wall synthesis. Cell Res. 17, 422-434 (2007).

51. $\mathrm{Li}$, J. et al. Mutation of rice $\mathrm{BC} 12 / \mathrm{GDD} 1$, which encodes a kinesin-like protein that binds to a GA biosynthesis gene promoter, leads to dwarfism with impaired cell elongation. Plant Cell 23, 628-640 (2011).

52. Grechkin, A. N. et al. Allene oxide synthase [athway in cereal roots: detection of novel oxylipin graminoxins. ChemistryOpen 7, 336-343 (2018).

\section{Acknowledgements}

The research described here was supported by the national key project for the research and development of China (2016YFD0100500) and the National Natural Science Foundation of China $(31772146,31972350)$. We thank professor Daowen Wang in Henan Agricultural University for his helpful discussions.

\section{Author contributions}

J.G. designed the experiment. Y.C. and Y.Y. performed most of the experiment. T.W. helped in the field trial experiments. S.W. and F.C. did the pollen sac cross-section experiment. G.Z. quantified fatty acids profiles. H.Y. and W.C. quantified the ABA contents. J.G., Y.Y., and Y.C. analyzed the data. J.G. and Y.Y. wrote the paper. All authors reviewed this manuscript.

\section{Competing interests}

The authors declare no conflict of interest.

\section{Additional information}

Supplementary information is available for this paper at https://doi.org/10.1038/s41467020-20133-z.

Correspondence and requests for materials should be addressed to J.-Y.G.

Peer review information Nature Communications thanks Chuanqing Sun, and the other, anonymous, reviewer(s) for their contribution to the peer review of this work. Peer reviewer reports are available.

Reprints and permission information is available at http://www.nature.com/reprints

Publisher's note Springer Nature remains neutral with regard to jurisdictional claims in published maps and institutional affiliations.

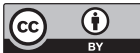

Open Access This article is licensed under a Creative Commons Attribution 4.0 International License, which permits use, sharing, adaptation, distribution and reproduction in any medium or format, as long as you give appropriate credit to the original author(s) and the source, provide a link to the Creative Commons license, and indicate if changes were made. The images or other third party material in this article are included in the article's Creative Commons license, unless indicated otherwise in a credit line to the material. If material is not included in the article's Creative Commons license and your intended use is not permitted by statutory regulation or exceeds the permitted use, you will need to obtain permission directly from the copyright holder. To view a copy of this license, visit http://creativecommons.org/ licenses/by/4.0\%

(C) The Author(s) 2020 